\title{
FLUORESCENCE BEHAVIOUR OF HIGHLY CONCENTRATED RHODAMINE 6G SOLUTIONS
}

\author{
A. PENZKOFER and W. LEUPACHER \\ Naturwissenschaftliche Fakultät II - Physik, Universität Regensburg, D-8400 Regensburg, Fed. Rep. Germany
}

Received 7 October 1986

Revised 23 January 1987

Accepted 27 January 1987

\begin{abstract}
The fluorescence quantum distributions $E(\lambda)$ and fluorescence quantum efficiencies $q_{\mathrm{F}}$ of rhodamine $6 \mathrm{G}$ in methanol and in water are measured for various concentrations up to the solubility limit. The fluorescence spectra are separated in monomer and dimer (ground-state dimer and closely spaced pair) contributions. The stimulated emission cross sections for the monomers and the dimers are resolved.
\end{abstract}

\section{Introduction}

The fluorescence spectra of highly concentrated dye solutions are scarcely investigated [1-3] since the fluorescence quantum efficiency reduces drastically [1-9] and reabsorption of fluorescence light distorts the frequency distribution $[10,11]$. The formation af aggregates as dimers [12], closely spaced pairs [13] and higher oligomers [12,14,15] is mainly studied by analyzing absorption changes.

For rhodamine $6 \mathrm{G}$ in methanol and water the absorption behaviour of highly concentrated solutions was studied in $[13,16]$. Rhodamine $6 \mathrm{G}$ in water forms stable ground-state dimers [16]. Rhodamine $6 \mathrm{G}$ in methanol has low tendency to form stable ground-state dimers [13]. At high concentrations the dye molecules come near together by random motion and they interact with one another (closely spaced pair formation [13]). For both stable ground-state dimers and closely spaced pairs the generic name dimers is used here.

For rhodamine $6 \mathrm{G}$ in methanol the dependence of the fluorescence quantum efficiency and the fluorescence lifetime on concentration was studied in [9]. Closely spaced pair fluorescence was resolved at high concentrations. The ground-state absorption recovery time versus concentration was measured in [17] and found to be approximately equal to the fluorescence lifetime. The short fluorescence lifetimes (e.g. $\tau_{\mathrm{F}} \simeq 2 \mathrm{ps}$, at $0.4 \mathrm{~mol} / \mathrm{l}$ ) and the equal values of fluorescence lifetime and ground-state absorption recovery time exclude triplet fluorescence and delayed singlet fluorescence caused by $S_{1}$-state repopulation from triplet states.

For rhodamine $6 \mathrm{G}$ in water no dimer fluorescence has been reported so far, since the monomer fluorescence dominates still at the highest possible dye concentration $\left(C_{\max }=0.027 \mathrm{~mol} / 1, \tau_{\mathrm{F}} \simeq 150\right.$ ps, see below).

In this paper the fluorescence spectra of rhodamine $6 \mathrm{G}$ in methanol and water are investigated at room temperature. The dye concentration is varied from very low values up to the solubility limit (methanol: $0.66 \mathrm{~mol} / \mathrm{l}$; water: $0.027 \mathrm{~mol} / \mathrm{l}$ ). From the measured fluorescence spectra the fluorescence quantum distributions $E(\lambda)$, the fluorescence quantum efficiencies $q_{\mathrm{F}}\left[\int_{\mathrm{em}} E(\lambda) \mathrm{d} \lambda=q_{\mathrm{F}}\right]$ and the monomer and dimer stimulated emission cross sections are determined. The resolved absorption and emission cross-section spectra of the closely spaced pairs of rhodamine $6 \mathrm{G}$ in methanol and of the stable ground-state dimers of rhodamine $6 \mathrm{G}$ in water are interpreted in terms of a dimer model that assumes different Franck-Con- 
don shifts between the $S_{0}$ and $S_{1}$ states of monomers, closely spaced pairs, and stable ground-state dimers.

\section{Experimental arrangement}

The fluorescence spectra are measured with the experimental setup shown in fig. 1a. A tungsten lamp (LS) is used as excitation source. The stabilized power supply of the tungsten lamp guarantees constant excitation of the sample. An interference filter (IF) restricts the excitation band-

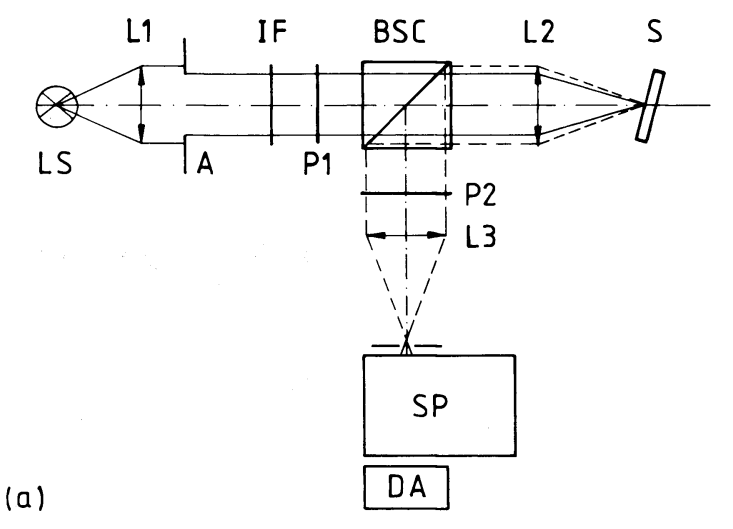

(b)
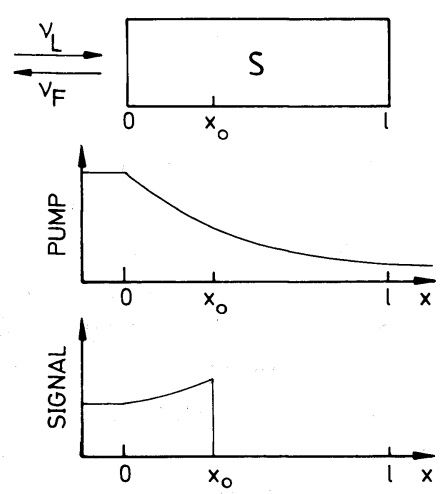

Fig. 1. (a) Experimental setup. LS, tungsten lamp; L1-L3, lenses; IF, interference filter; BSC, $50 \%$ beam splitting cube; $\mathrm{S}$, sample; SP, spectrograph; DA, diode array system. P1 and P2, polarizer sheets (included in fluorescence depolarization analysis). (b) Pump light attenuation and fluorescence signal attenuation (generated at $x_{0}$ ) in sample. Drawings illustrate derivation of eq. (2). width close to the $S_{0}-S_{1}$ absorption peak (slightly shifted to short-wavelength side). The pump light is focused to the sample $\mathrm{S}$ with lens L2. The fluorescence emission in backward direction is gathered by lens L2 and directed to the spectrometer SP by a broad-band 50 percent beam splitting cube (BSC) and lens L3. The dispersed fluorescence spectrum is registered by a diode array system (Tracor DARRS system) and the data are transfered to a computer for analysis. For fluorescence depolarization analysis two polarizer sheets $\mathrm{P} 1$ and $\mathrm{P} 2$, are inserted in the experimental system, one in the excitation path between IF and BSC and one in the detection path between BSC and L3. The fluorescence signal is independent of molecular reorientation if the polarizer sheets are oriented under an angle of $\phi=\arctan \left(2^{1 / 2}\right)=$ $54.74^{\circ}$ (e.g. P1 vertical, P2 at angle $\phi=54.74^{\circ}$ to the vertical axis) $[18,19]$. The fluorescence depolarization is obtained by orienting alternately both polarizers parallel (P1 and P2 vertical) and perpendicular (P1 vertical, $\mathrm{P} 2$ horizontal).

\section{Fluorescence parameter extraction}

The diode array detection system measures the spectral photon distribution $S_{\mathrm{m}}(\lambda)$ behind the spectrometer within a time duration $\Delta t$ (unit: counts $\mathrm{nm}^{-1}$, proportional to photons $\mathrm{nm}^{-1}$ ). The fluorescence signal $S_{\mathrm{E}}(\lambda)$ emitted from the sample $S$ within the acceptance angle $\Delta \Omega$ of lens L2 is calculated by taking care of the spectral transmission $T_{\mathrm{BSC}}$ of the beam splitter cube BSC, of the spectral transmission $T_{\mathrm{SP}}$ of the spectrometer SP and of the spectral sensitivity $S_{\mathrm{DA}}$ (counts/photon) of the diode array DA. The relation between $S_{\mathrm{E}}(\lambda)$ and $S_{\mathrm{m}}(\lambda)$ is

$S_{\mathrm{E}}(\lambda)=\frac{S_{\mathrm{m}}(\lambda)}{T_{\mathrm{BSC}}(\lambda) T_{\mathrm{SP}}(\lambda) S_{\mathrm{DA}}(\lambda)}$.

The intrinsic signal $S_{\mathrm{I}}(\lambda)$ inside the sample is different from the external signal $S_{\mathrm{E}}(\lambda)$ outside the sample because of reabsorption of fluorescence light along the path from the position of generation towards the exit window. The situation is illustrated in fig. 1b. At depth $x_{0}$ inside the sample the pump power $P$ is reduced to $P\left(x_{0}\right)=$ 
$P(0) \exp \left(-N \sigma_{\mathrm{L}} x_{0}\right)\left(N=N_{\mathrm{A}} C\right.$ is the number density of dye molecules, $N_{\mathrm{A}}=6.022045 \times 10^{23} \mathrm{~mol}^{-1}$ Avogadro's constant, $C$ concentration, $\sigma_{\mathrm{L}}$ absorption cross section of dye molecules at pump light wavelength $\lambda_{\mathrm{L}}$ ). At position $x_{0}$ the contribution to the intrinsic fluorescence signal is

$$
\begin{aligned}
\mathrm{d} S_{\mathrm{I}}(\lambda) / \mathrm{d} x & =-\operatorname{const}(\lambda) \mathrm{d} P / \mathrm{d} x \\
& =\operatorname{const}(\lambda) P(0) \exp \left(-N \sigma_{\mathrm{L}} x_{0}\right) N \sigma_{\mathrm{L}}
\end{aligned}
$$

and the contribution to the external signal is

$$
\begin{aligned}
\mathrm{d} S_{\mathrm{E}}(\lambda) / \mathrm{d} x= & (1-R) \exp \left[-N \sigma(\lambda) x_{0}\right] \frac{\mathrm{d} S_{\mathrm{I}}(\lambda)}{\mathrm{d} x} \\
= & (1-R) \operatorname{const}(\lambda) P(0) \\
& \times \exp \left\{-N\left[\sigma_{\mathrm{L}}+\sigma(\lambda)\right] x_{0}\right\} N \sigma_{\mathrm{L}} .
\end{aligned}
$$

$R$ is the reflectivity of fluorescence light at the window. The total intrinsic fluorescence signal is

$S_{\mathrm{I}}(\lambda)=\int_{0}^{l} \mathrm{~d} S_{\mathrm{I}}(\lambda)=\operatorname{const}(\lambda) P(0)\left(1-T_{\mathrm{L}}\right)$.

$T_{\mathrm{L}}=\exp \left(-N \sigma_{\mathrm{L}} l\right)$ is the pump pulse transmission. The total external fluorescence signal is

$$
\begin{aligned}
S_{\mathrm{E}}(\lambda)= & \int_{0}^{l} \mathrm{~d} S_{\mathrm{E}}(\lambda) \\
= & (1-R) \operatorname{const}(\lambda) \\
& \times P(0)\left\{1-\exp \left[-N\left(\sigma_{\mathrm{L}}+\sigma(\lambda)\right) l\right]\right\} \sigma_{\mathrm{L}} \\
& \times\left[\sigma_{\mathrm{L}}+\sigma(\lambda)\right]^{-1} \\
= & (1-R) \operatorname{const}(\lambda) \\
& \times P(0)\left\{1-T_{\mathrm{L}}^{\left[\sigma_{\mathrm{L}}+\sigma(\lambda)\right] / \sigma_{\mathrm{L}}}\right\} \sigma_{\mathrm{L}} \\
& \times\left[\sigma_{\mathrm{L}}+\sigma(\lambda)\right]^{-1} .
\end{aligned}
$$

The relation between internal and external fluorescence signal becomes

$S_{\mathrm{I}}(\lambda)=\frac{\sigma_{\mathrm{L}}+\sigma(\lambda)}{\sigma_{\mathrm{L}}(1-R)} \frac{1-T_{\mathrm{L}}}{1-T_{\mathrm{L}}^{\left[\sigma_{\mathrm{L}}+\sigma(\lambda)\right] / \sigma_{\mathrm{L}}}} S_{\mathrm{E}}(\lambda)$.

In the analysis reemission of absorbed fluorescence light within the acceptance angle $\Delta \Omega_{\mathrm{I}}=$ $\Delta \Omega / \eta_{\mathrm{F}}^{2}\left(\eta_{\mathrm{F}}\right.$ is the refractive index of the solution at fluorescence wavelength $\lambda$ ) is neglected since $\Delta \Omega_{\mathrm{I}}$ is small compared to $4 \pi$ and at high concentration the fluorescence quantum efficiency is low.

The fluorescence quantum distribution $E(\lambda)$ is defined as the ratio of total intrinsic fluorescence signal integrated over the full solid angle $4 \pi$ $\left[S_{\mathrm{I}, \mathrm{t}}(\lambda)=S_{\mathrm{I}}(\lambda) 4 \pi / \Delta \Omega_{\mathrm{I}}\right]$ to the absorbed pump photons $\left[n_{\text {abs }}=P(0) \Delta t\left(1-T_{\mathrm{L}}\right) / h \nu_{\mathrm{L}}, \Delta t\right.$ is the integration time of the diode array system] leading to

$E(\lambda)=\frac{4 \pi \eta_{\mathrm{F}}^{2}}{\Delta \Omega} \frac{S_{\mathrm{I}}(\lambda) h \nu_{\mathrm{L}}}{P(0)\left[1-T_{\mathrm{L}}\right] \Delta \mathrm{t}}$.

The fluorescence quantum efficiency $q_{\mathrm{F}}$ (the ratio of total number of intrinsic fluorescence photons to absorbed pump light photons) is given by

$q_{\mathrm{F}}=\int_{\mathrm{em}} E(\lambda) \mathrm{d} \lambda$.

The integration extends over the $S_{1}-S_{0}$ fluorescence band. Often a normalized fluorescence quantum distribution $\tilde{E}(\lambda)$ is used which is defined by $\tilde{E}(\lambda)=E(\lambda) / q_{\mathrm{F}}$, i.e., $\int_{\mathrm{em}} \tilde{E}(\lambda) \mathrm{d} \lambda=1$.

In the experiments $E(\lambda)$ and $q_{\mathrm{F}}$ are determined by calibration to the fluorescence signal of a reference substance of known quantum efficiency $q_{\mathrm{R}}$ in order to get rid of geometrical factors and absolute energy measurements. In our case $10^{-5}$ molar rhodamine $6 \mathrm{G}$ in methanol is used as reference $\left(q_{\mathrm{R}} \simeq 0.9\right.$ [6]). The quantum efficiency is found by use of relation (4)

$$
q_{\mathrm{F}} / q_{\mathrm{R}}=\int_{\mathrm{em}} E(\lambda) \mathrm{d} \lambda / \int_{\mathrm{em}} E_{\mathrm{R}}(\lambda) \mathrm{d} \lambda
$$

and eq. (3):

$$
q_{\mathrm{F}}=\frac{\eta_{\mathrm{F}}^{2} \int_{\mathrm{em}} S_{\mathrm{I}}(\lambda) \mathrm{d} \lambda}{\eta_{\mathrm{R}}^{2} \int_{\mathrm{em}} S_{\mathrm{I}, \mathrm{R}}(\lambda) \mathrm{d} \lambda} \frac{1-T_{\mathrm{L}, \mathrm{R}}}{1-T_{\mathrm{L}}} q_{\mathrm{R}}
$$

The fluorescence quantum distribution is given by

$$
E(\lambda)=\frac{\eta_{\mathrm{F}}^{2} S_{\mathrm{I}}(\lambda)}{\eta_{\mathrm{R}}^{2} \int_{\mathrm{em}} S_{\mathrm{I}, \mathrm{R}}(\lambda) \mathrm{d} \lambda} \frac{1-T_{\mathrm{L}, \mathrm{R}}}{1-T_{\mathrm{L}}} q_{\mathrm{R}},
$$

$S_{\mathrm{I}}(\lambda)$ and $S_{\mathrm{I}, \mathrm{R}}(\lambda)$ are related to the measured quantities $S_{\mathrm{m}}(\lambda)$ and $S_{\mathrm{m}, \mathrm{R}}(\lambda)$ by eqs. (1) and (2).

The fluorescence anisotropy $r(\lambda)$ is defined by 
$[18,19]$

$$
\begin{aligned}
r(\lambda) & =\frac{E_{\|}(\lambda)-E_{\perp}(\lambda)}{E_{\|}(\lambda)+2 E_{\perp}(\lambda)} \\
& =\frac{S_{\mathrm{I}, \mathrm{l}}(\lambda)-S_{\mathrm{I}, \perp}(\lambda)}{S_{\mathrm{I}, \mathrm{ll}}(\lambda)+2 S_{\mathrm{I}, \perp}(\lambda)},
\end{aligned}
$$

$E_{\|}$and $E_{\perp}$ are the fluorescence quantum distributions for parallel and perpendicular oriented polarizers, respectively. $S_{\mathrm{I}, \|}$ is the intrinsic fluorescence signal for parallel oriented polarizers and $S_{\mathrm{I}, \perp}$ is the intrinsic fluorescence signal for perpendicular oriented polarizers. If no molecular reorientation of the excited molecules occurs within the fluorescence lifetime $\tau_{\mathrm{F}}$, then the anisotropy is $r=0.4$ for parallel orientation of the absorption and emission transition dipole moment, and $r=-0.2$ for perpendicular orientation of the absorption and emission dipole moment $[19,20]$. In case of fast reorientation of the excited molecules within the fluorescence lifetime, $\tau_{\mathrm{F}}$, it is $r=0$. At high dye concentration fast energy transfer $[14,12,9]$ depolarizes the fluorescence emission $(r \rightarrow 0)$ even in highly viscous solvents. If fluorescence anisotropy is present, it is necessary to use two polarizers under an angle of $54.74^{\circ}$ (see above) in order to get rid of orientational effects (otherwise eq. (3) is inexact, since $S_{\mathrm{I}}(\lambda)$ becomes dependent on observation direction).

\section{Results}

The measured fluorescence quantum distributions $E(\lambda)$ of rhodamine $6 \mathrm{G}$ in methanol and of rhodamine $6 \mathrm{G}$ in water are shown by the solid curves in figs. 2 and 3 , respectively. The fluorescence quantum efficiencies $q_{\mathrm{F}}$ are shown in fig. 4 for rhodamine $6 \mathrm{G}$ in methanol and in fig. 5 for rhodamine $6 \mathrm{G}$ in water (triangles).

In case of rhodamine $6 \mathrm{G}$ in methanol, $E(\lambda)$ and $q_{\mathrm{F}}$ are independent of concentration up to about $5 \times 10^{-3} \mathrm{~mol} / \mathrm{l}$. At higher concentration $E(\lambda)$ and $q_{\mathrm{F}}$ decrease strongly with increasing concentration. For $C>0.1 \mathrm{~mol} / 1$ the quantum efficiency levels off to a limiting value of about $q_{\mathrm{F}} \simeq 6.5 \times 10^{-4}$ at $0.62 \mathrm{~mol} / 1$. The fluorescence spectra change their shape in the high-concentra-

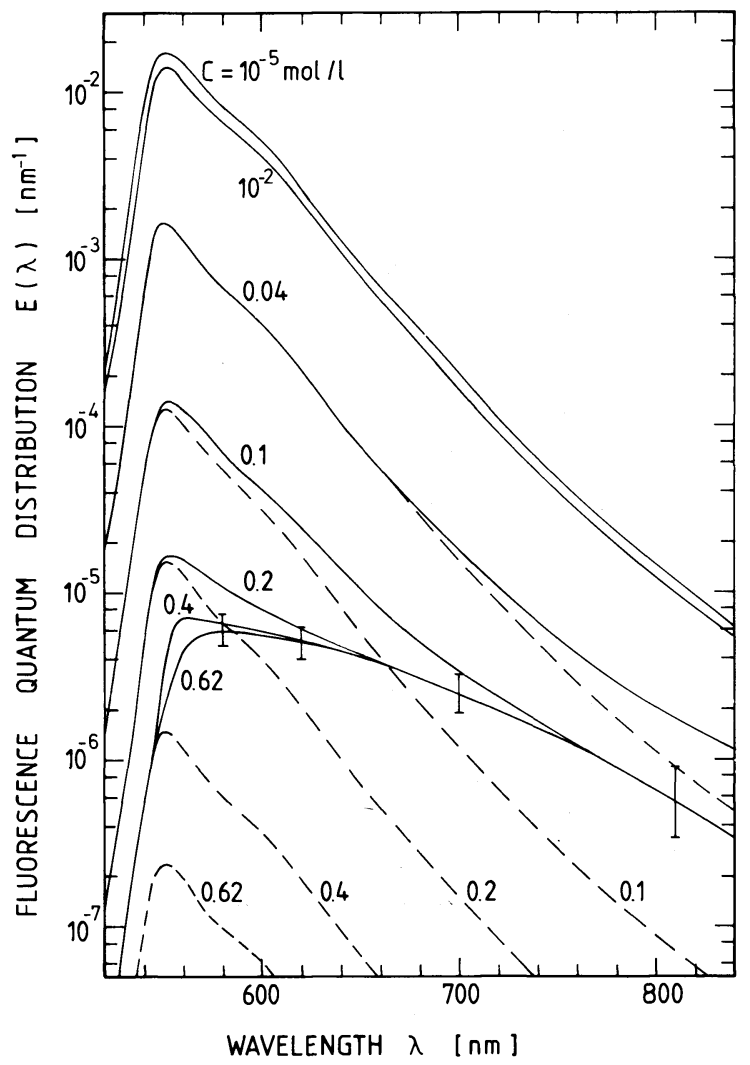

Fig. 2. Fluorescence quantum distribution $E(\lambda)$ of rhodamine 6G in methanol. Solid curves, measured $E(\lambda)$ distributions for various concentrations. Dashed curves, calculated monomeric contributions $E_{\mathrm{M}}(\lambda, C)$.

tion region $(C \geqslant 0.1 \mathrm{~mol} / \mathrm{l})$. The short-wavelength part of the spectra continues to decrease with concentration while the long-wavelength part remains practically unchanged. The concentration dependence of the fluorescence lifetime $\tau_{F}$ of rhodamine $6 \mathrm{G}$ in methanol was measured recently with a streak-camera [9] and the results are included in fig. 4 (open circles, dashed curve gives least-square fit). In [9] it was shown that the decrease of $\tau_{\mathrm{F}}$ and $q_{\mathrm{F}}$ is due to Förster-type excitation transfer from monomers to weakly fluorescing closely spaced pairs [13] which are formed randomly at high concentration.

In case of rhodamine $6 \mathrm{G}$ in water the fluorescence quantum distribution $E(\lambda)$ and the fluorescence quantum efficiency $q_{\mathrm{F}}$. are practically 


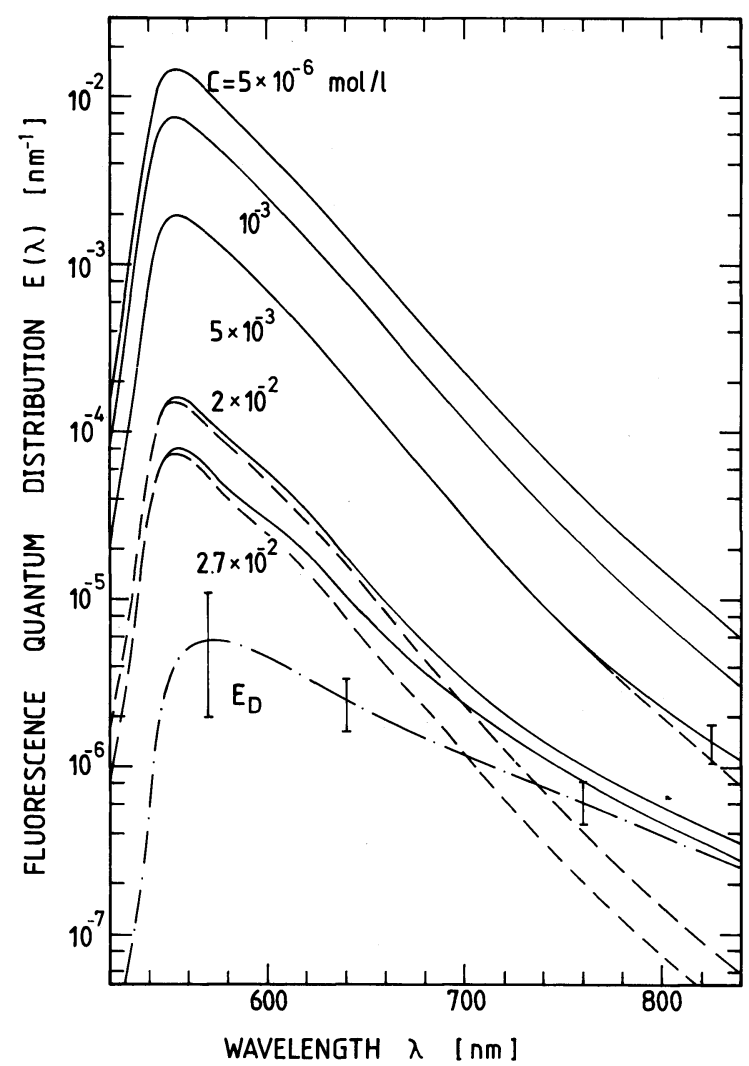

Fig. 3. Fluorescence quantum distribution $E(\lambda)$ of rhodamine $6 \mathrm{G}$ in $\mathrm{H}_{2} \mathrm{O}$. Solid curves, measured $E(\lambda)$ distributions for various concentrations. Dashed curves, calculated monomeric contributions $E_{\mathrm{M}}(\lambda, C)$. Dashed-dotted curve, extracted dimer fluorescence quantum distribution $E_{\mathrm{D}}(\lambda)=E_{\mathrm{D}}(\lambda$,

$$
\left.x_{\mathrm{D}} \rightarrow 1\right) \text {. }
$$

constant for $C<5 \times 10^{-5} \mathrm{~mol} / \mathrm{l}$. Above $10^{-3}$ mol/l $q_{\mathrm{F}}$ decreases strongly and $E(\lambda)$ decreases more severely at short wavelengths than at long wavelengths. At the solubility limit of $0.027 \mathrm{~mol} / 1$ the fluorescence quantum efficiency is $q_{\mathrm{F}} \simeq 4.5 \times$ $10^{-3}$. The fluorescence lifetime was measured with a streak camera and found to be $\tau_{\mathrm{F}} \simeq 150 \mathrm{ps}$ at $C_{\max }=0.027 \mathrm{~mol} / 1$ (arrangement similar to fig. 1 of ref. [9]). The decrease of $q_{\mathrm{F}}$ and $E(\lambda)$ is thought to be due to Förster-type transfer of excitation energy from monomers to weakly fluorescing stable ground-state dimers $[9,13]$. The short fluorescence lifetime excludes triplet contributions to the fluorescence signal.

The fluorescence anisotropy is analyzed for

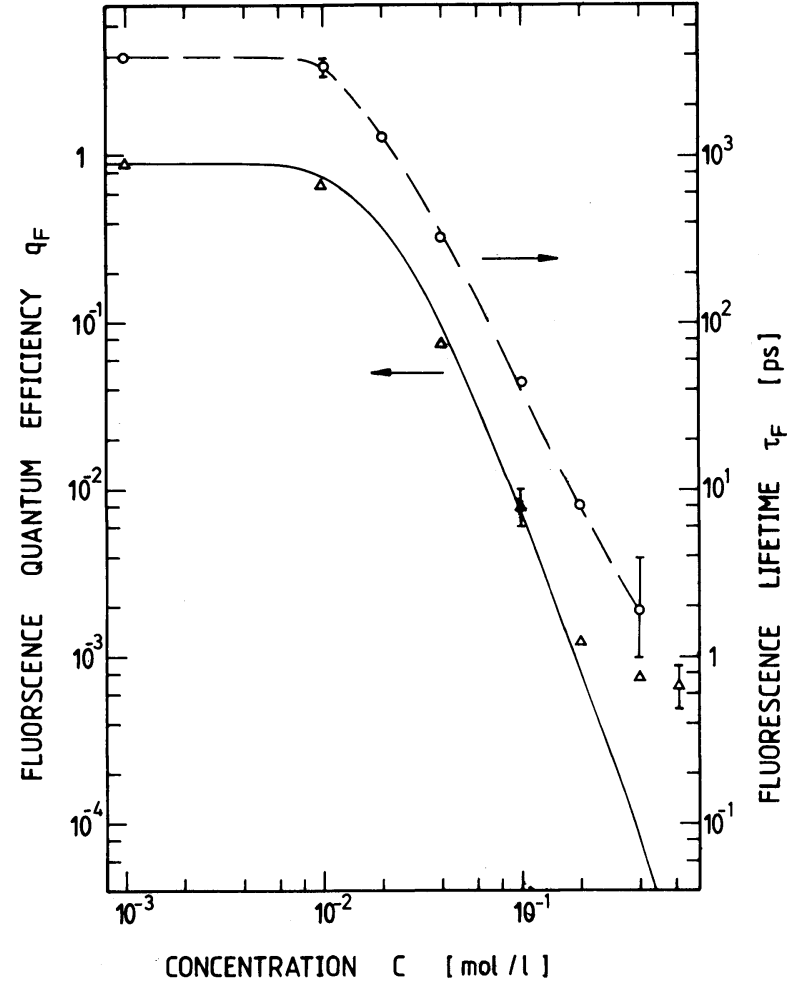

Fig. 4. Fluorescence quantum efficiency $q_{\mathrm{F}}$ versus concentration $C$ for rhodamine $6 \mathrm{G}$ in methanol (triangles are experimental values, the solid curve is calculated by use of eq. (10)). Fluorescence lifetimes $\tau_{F}$ (open circles and dashed line) are included (from [9]).

rhodamine $6 \mathrm{G}$ in methanol. Complete fluorescence depolarization $r(\mathrm{C})=0$ is observed for all concentrations $\left(10^{-5} \mathrm{~mol} / 1 \leq C \leq 0.62 \mathrm{~mol} / \mathrm{l}\right)$ within the experimental accuracy. At low concentrations $C<5 \times 10^{-3} \mathrm{~mol} / 1$ the fluorescence lifetime $\left(\tau_{\mathrm{F}}=3.9 \mathrm{~ns}\right)$ is long compared to the molecular reorientation time $\left(\tau_{\text {or }} \approx 100 \mathrm{ps}[20-22]\right)$ leading to an anisotropy factor of $r=0$. In a medium concentration region $\left(2 \times 10^{-2} \mathrm{~mol} / 1<\right.$ $C<0.2 \mathrm{~mol} / \mathrm{l}) \tau_{\mathrm{F}}$ becomes comparable to $\tau_{\text {or }}$ or shorter than $\tau_{\mathrm{or}}$. The Förster-type excitation energy transfer from monomer to monomer depolarizes the fluorescence signal. At high concentrations $(C \geq 0.2 \mathrm{~mol} / \mathrm{l})$ the closely-spaced pair fluorescence dominates $\left(\tau_{\mathrm{F}} \approx \tau_{\mathrm{D}}<\tau_{\mathrm{or}}\right)$. In this region the average distance between closely spaced pairs becomes less than the Förster-transfer radius $R_{\text {o }}$ (see [9]) and the excitation energy transfer rate 


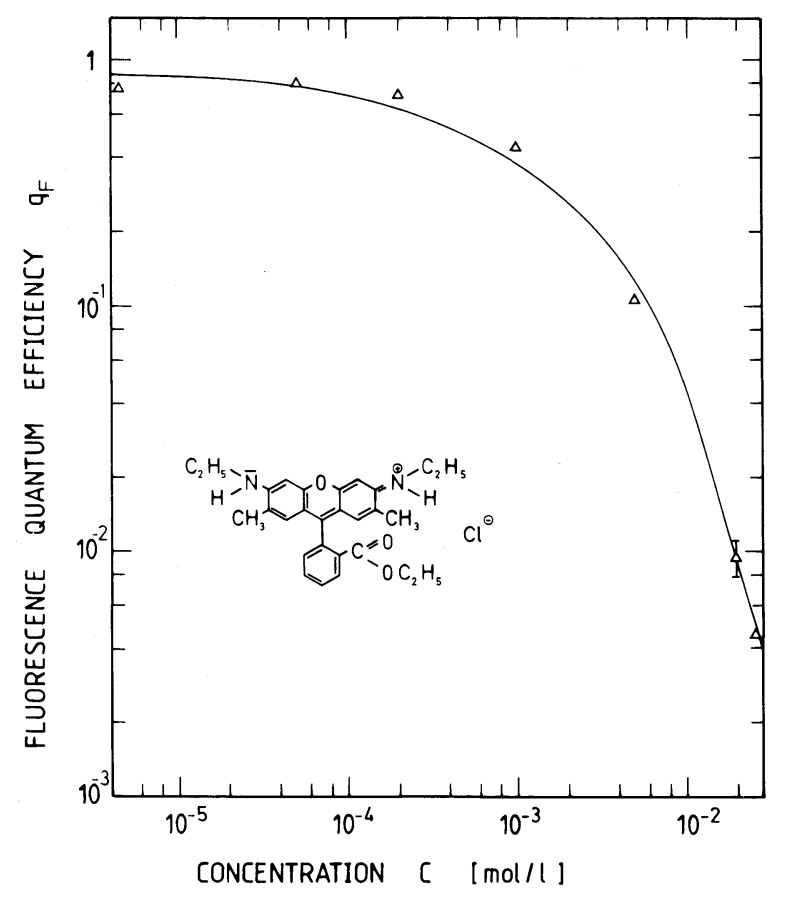

Fig. 5. Fluorescence quantum efficiency $q_{\mathrm{F}}$ of rhodamine $6 \mathrm{G}$ in $\mathrm{H}_{2} \mathrm{O}$. Triangles are experimental values. The curve is calculated by use of eq. (10). The structure formula of rhodamine $6 \mathrm{G}$ is inserted.

between closely spaced pairs is faster than the fluorescence decay rate resulting in depolarized emission.

In case of rhodamine $6 \mathrm{G}$ in water the fluorescence lifetime at the highest possible concentration $(C=0.027 \mathrm{~mol} / \mathrm{l})$ is about $150 \mathrm{ps}$. The molecular reorientation time is $\tau_{\text {or }} \simeq 170$ ps [22]. At low concentrations fluorescence depolarization occurs because of $\tau_{\mathrm{F}}>\tau_{\mathrm{or}}$. Towards the solubility limit the depolarization is enhanced by excitation energy migration.

\section{Monomeric and dimeric contributions to $E(\lambda)$ and $q_{F}$}

In the following $E(\lambda)$ and $q_{\mathrm{F}}$ are separated into monomeric and dimeric contributions. As analyzed in [13] two components are formed at elevated concentrations in methanolic rhodamine 6G (monomers and closely spaced pairs) and aqueous rhodamine 6G (monomers and groundstate dimers) solutions. The mole fraction $x_{\mathrm{D}}$ of molecules forming these dimers was determined as a function of concentration by analyzing the absorption changes with concentration [13] and the result is depicted in fig. 6 .

The fluorescence quantum distribution $E(\lambda)$ and the fluorescence quantum efficiency $q_{\mathrm{F}}$ may be separated into monomeric and dimeric parts:

$$
\begin{aligned}
& E(\lambda, C)=E_{\mathrm{M}}(\lambda, C)+E_{\mathrm{D}}(\lambda, C), \\
& q_{\mathrm{F}}(C)=q_{\mathrm{M}}(C)+q_{\mathrm{D}}(C),
\end{aligned}
$$

$E_{\mathrm{M}}(\lambda, C)$ and $q_{\mathrm{M}}(C)$ represent the fluorescence part emitted from monomers, while $E_{\mathrm{D}}(\lambda)$ and $q_{\mathrm{D}}$ describe the fluorescence part emitted from dimers (ground-state dimers or closely-spaced pairs).

The decrease of monomer fluorescence quantum efficiency $q_{\mathrm{M}}(C)$ and fluorescence quantum distribution $E_{\mathrm{M}}(\lambda, C)$ is caused by Förster-type energy transfer (electric dipole-electric dipole interaction) to dimers (quenching centers, see [9]) and is given by [9]

$$
\begin{aligned}
& q_{\mathrm{M}}(C) \simeq\left(1-x_{\mathrm{D}}\right) \frac{q_{\mathrm{F}}(0)}{1+x_{\mathrm{D}}\left(C / C_{0}\right)^{2}}, \\
& E_{\mathrm{M}}(\lambda, C)=\frac{q_{\mathrm{M}}(C)}{q_{\mathrm{F}}(0)} E(\lambda, 0),
\end{aligned}
$$

where $C_{0}$ is the critical transfer concentration. In eq. (10) energy back-transfer from dimers to monomers is neglected since the relaxed excited dimer states lie below the relaxed excited monomer states (overlap integral between dimer fluorescence spectrum and monomer absorption spectrum is reduced as is seen in figs. 2, 3, 7 and 8 , for inclusion of energy back-transfer see [9]).

The $C_{0}$-values of rhodamine $6 \mathrm{G}$ in methanol and in water are found by fitting eq. (10) to the experimental $q_{\mathrm{F}}$-values at $C=0.1 \mathrm{~mol} / 1$ and $C=$ $0.02 \mathrm{~mol} / 1$, respectively. The results are $C_{0}=$ $4.5 \times 10^{-3} \mathrm{~mol} / 1$ (transfer radius $R_{0}=$ $\left.\left[3 / 4 \pi N_{\mathrm{A}} C_{0}\right]^{1 / 3}=4.45 \mathrm{~nm}\right)$ in case of solvent methanol and $C_{0}=5.6 \times 10^{-3} \mathrm{~mol} / 1\left(R_{0}=4.14\right.$ $\mathrm{nm}$ ) for the aqueous solution.

The solid curves in figs. 4 and 5 present the theoretical $q_{\mathrm{M}}(C)$ curves of eq. (10). In case of rhodamine $6 \mathrm{G}$ in methanol, $q_{\mathrm{M}}(C)$ continues to 


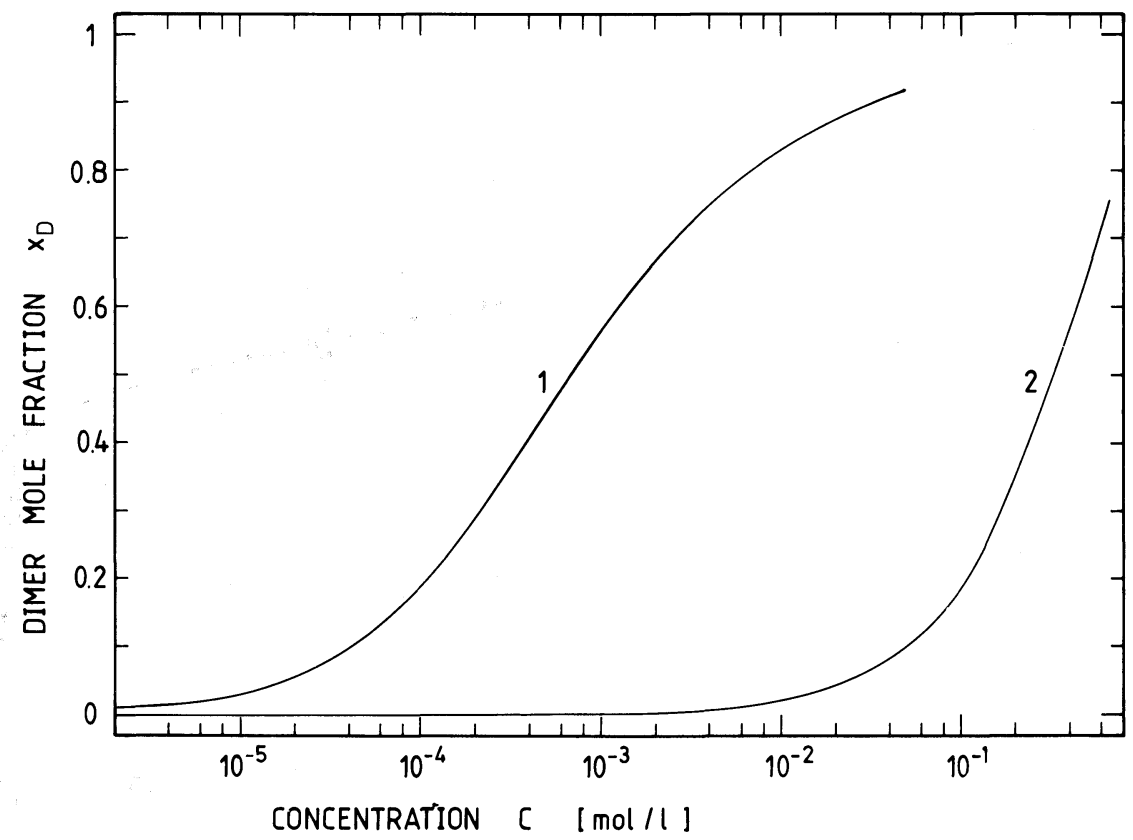

Fig. 6. Fraction $x_{\mathrm{D}}$ of molecules in dimer state (from [13]). Curve 1: rhodamine $6 \mathrm{G}$ in water $\left(x_{\mathrm{D}} / 2\right.$ is mole fraction of stable ground-state dimers). Curve 2: rhodamine $6 \mathrm{G}$ in methanol $\left(x_{\mathrm{D}} / 2\right.$ is mole fraction of closely spaced pairs).

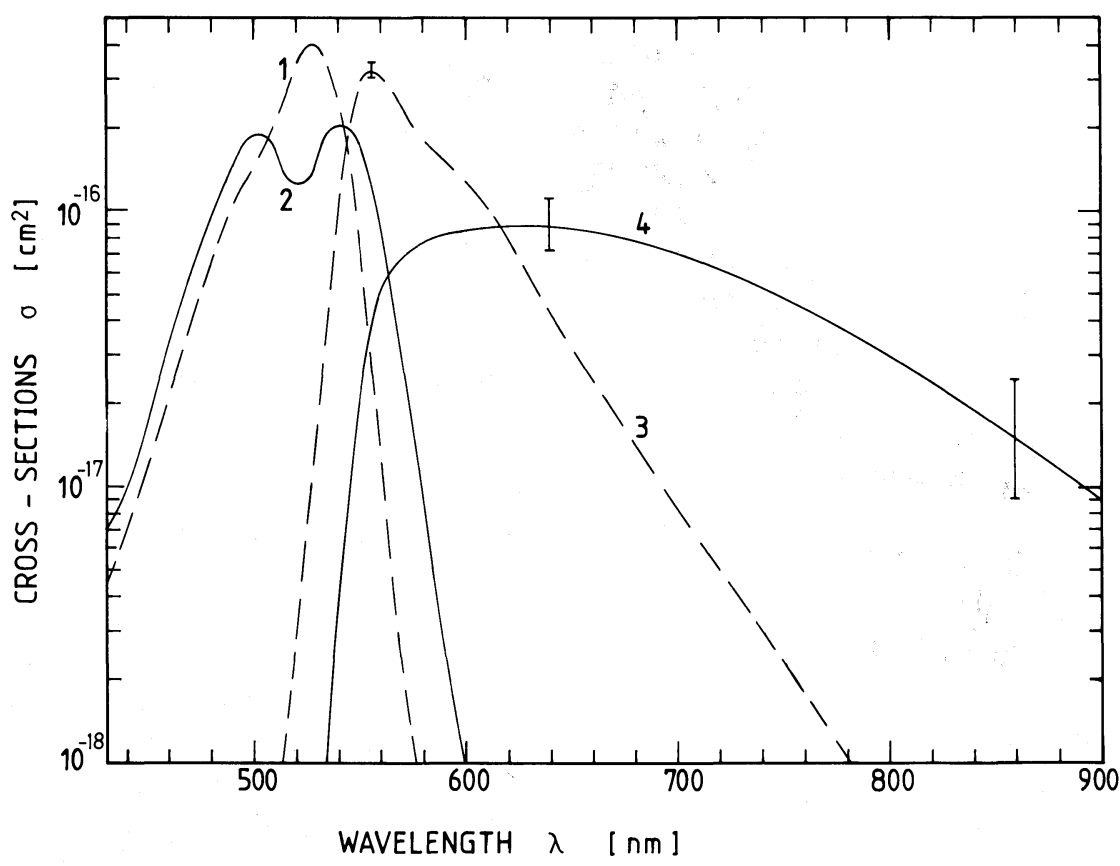

Fig. 7. Absorption and stimulated emission cross-section spectra $\sigma$ of monomers and closely spaced pairs of rhodamine $6 \mathrm{G}$ in methanol. Curve 1, $\sigma_{\mathrm{abs}, \mathrm{M}}(\lambda)$; curve 2, $\sigma_{\mathrm{abs}, \mathrm{D}}(\lambda)$; curve 3, $\sigma_{\mathrm{em}, \mathrm{M}}(\lambda)$; curve $4, \sigma_{\mathrm{em}, \mathrm{D}}(\lambda)$. 


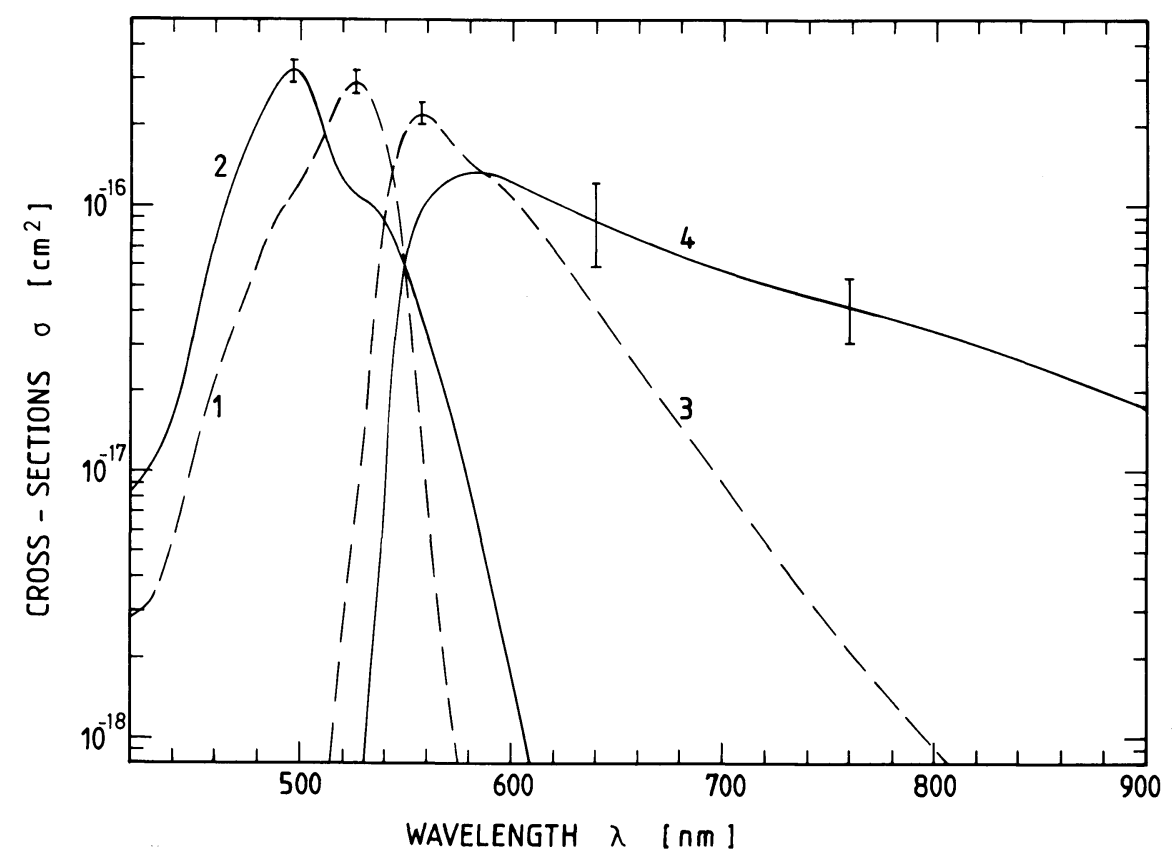

Fig. 8. Absorption and stimulated emission cross-section spectra $\sigma$ of monomers and ground-state dimers of rhodamine $6 \mathrm{G}$ in water. Curve $1, \sigma_{\mathrm{abs}, \mathrm{M}}(\lambda)$; curve 2, $\sigma_{\mathrm{abs}, \mathrm{D}}(\lambda)$; curve $3, \sigma_{\mathrm{em}, \mathrm{M}}(\lambda)$; curve $4, \sigma_{\mathrm{em}, \mathrm{D}}(\lambda)$.

decrease strongly for $C \geq 0.2 \mathrm{~mol} / 1$ while the experimental $q_{\mathrm{F}}$-values level off to a slight decrease. The difference between the experimental $q_{\mathrm{F}}$-values and the theoretical $q_{\mathrm{M}}$ curve indicates the dimer contribution $q_{\mathrm{D}}=q_{\mathrm{F}}-q_{\mathrm{M}}$ (eq. (9)). For rhodamine $6 \mathrm{G}$ in water no difference between the experimental $q_{\mathrm{F}}$ points and the theoretical $q_{\mathrm{M}}$ curve is resolvable within experimental accuracy. This fact indicates that up to the solubility limit the fluorescence emitted from dimers is small compared to the fluorescence emitted from monomers.

The monomeric contribution to the fluorescence quantum distribution (eq. (11)) is depicted by the dashed curves in figs. 2 and 3 for rhodamine $6 \mathrm{G}$ in methanol and water, respectively. The differences $E_{\mathrm{D}}(\lambda, C)=E(\lambda, C)-$ $E_{\mathrm{M}}(\lambda, C)$ represent the fluorescence emission from excited dimer states (states are excited either directly by light absorption or indirectly by energy transfer from excited monomers).

For 0.62 molar rhodamine $6 \mathrm{G}$ in methanol the monomer fluorescence contribution is negligibly small and the measured fluorescence quantum dis- tribution represents the closely spaced pair fluorescence quantum distribution $E_{\mathrm{D}}(\lambda)=E_{\mathrm{D}}(\lambda$, $\left.x_{\mathrm{D}} \rightarrow 1\right)$. This dimer fluorescence distribution is spectrally broader $\left(\Delta \tilde{\nu}_{\mathrm{D}} \simeq 3500 \mathrm{~cm}^{-1}\right.$ FWHM) than the monomer fluorescence distribution $\left(\Delta \tilde{\nu}_{M}\right.$ $\simeq 1700 \mathrm{~cm}^{-1}$ ). The maximum position of the dimer distribution is shifted about $1000 \mathrm{~cm}^{-1}$ to lower frequencies. The closely spaced pair fluorescence quantum efficiency is $q_{\mathrm{D}}=\int_{\mathrm{em}} E_{\mathrm{D}}(\lambda) \mathrm{d} \lambda \simeq$ $8.5 \times 10^{-4}$.

For the 0.027 molar aqueous rhodamine $6 \mathrm{G}$ solution (maximum concentration $C_{\max }$, solubility limit at room temperature) the monomer fluorescence quantum distribution $E_{\mathrm{M}}(\lambda)$ still dominates $E(\lambda)$, especially at short wavelengths. But the dimer contribution $E_{\mathrm{D}}\left(\lambda, C_{\max }\right)=E\left(\lambda, C_{\max }\right)-$ $E_{\mathrm{M}}\left(\lambda, C_{\text {max }}\right)$ is clearly resolved. $E_{\mathrm{D}}\left(\lambda, C_{\max }\right)$ is practically identical to $E_{\mathrm{D}}(\lambda)=E_{\mathrm{D}}\left(\lambda, x_{\mathrm{D}} \rightarrow 1\right)$ since nearly all monomer excitation is transferred to dimers $\left[q_{\mathrm{F}}\left(C_{\max }\right) \simeq q_{\mathrm{M}}\left(C_{\max }\right) \simeq 0.0045\right] . E_{\mathrm{D}}(\lambda)$ is depicted by the dashed-dotted curve in fig. 3 . The accuracy of $E_{\mathrm{D}}(\lambda)$ is somewhat reduced at the wavelength region of maximum emission because the difference between two nearly equal 
quantities has to be formed. $E_{\mathrm{D}}(\lambda)$ represents the fluorescence emission from excited stable groundstate dimers. The spectral width of $E_{\mathrm{D}}$ is $\Delta \tilde{\nu}_{\mathrm{D}} \simeq$ $2500 \mathrm{~cm}^{-1}$ (monomer: $\Delta \tilde{\nu}_{\mathrm{M}} \simeq 1400 \mathrm{~cm}^{-1}$ ) and the peak position is shifted about $700 \mathrm{~cm}^{-1}$ to the long-wavelength side. The dimer fluorescence efficiency is $q_{\mathrm{D}}=\int_{\mathrm{em}} E_{\mathrm{D}}(\lambda) \mathrm{d} \lambda \simeq 6 \times 10^{-4}$.

\section{Monomeric and dimeric stimulated emission cross sections}

Knowing the fluorescence quantum distribution $E_{\mathrm{M}}(\lambda)=E(\lambda, C \rightarrow 0)$ and $E_{\mathrm{D}}(\lambda)=E_{\mathrm{D}}(\lambda$, $\left.x_{\mathrm{D}} \rightarrow 1\right)$ and the monomer and dimer absorption cross-section spectra $\sigma_{\text {abs, } M}(\lambda)$ and $\sigma_{\text {abs,D }}(\lambda)$, the stimulated emission cross-section spectra $\sigma_{\mathrm{em}, \mathrm{M}}(\lambda)$ and $\sigma_{\mathrm{em}, \mathrm{D}}(\lambda)$ of the monomers $(\mathrm{i}=\mathrm{M})$ and dimers $(i=D)$ may be calculated by use of the formulae [23]

$\sigma_{\mathrm{em}, \mathrm{i}}(\lambda)=\frac{\lambda^{4} E_{\mathrm{i}}(\lambda)}{8 \pi \eta_{\mathrm{F}}^{2} c_{0} \tau_{\mathrm{rad}, \mathrm{i}} q_{\mathrm{i}}}=\frac{\lambda^{4} \tilde{E}_{\mathrm{i}}(\lambda)}{8 \pi \eta_{\mathrm{F}}^{2} c_{0} \tau_{\mathrm{rad}, \mathrm{i}}}$,

and $[24,25]$

$$
\begin{aligned}
\frac{1}{\tau_{\mathrm{rad}, \mathrm{i}}}= & \frac{8 \pi \eta_{\mathrm{F}}^{3} c_{0}}{\eta_{\mathrm{A}}} \frac{\int_{\mathrm{em}} E_{\mathrm{i}}(\lambda) \lambda \mathrm{d} \lambda}{\int_{\mathrm{em}} E_{\mathrm{i}}(\lambda) \lambda^{4} \mathrm{~d} \lambda} \\
& \times \int_{\mathrm{abs}} \frac{\sigma_{\mathrm{abs}, \mathrm{i}}(\lambda)}{\lambda} \mathrm{d} \lambda,
\end{aligned}
$$

$c_{0}$ is the vacuum light velocity and $\eta_{\mathrm{A}}$ the average refractive index in the $S_{0}-S_{1}$ absorption band. The integrations extend over the $S_{1}-S_{0}$ fluorescence band $\left[E_{\mathrm{i}}(\lambda)\right]$ and the $\mathrm{S}_{0}-\mathrm{S}_{1}$ absorption band $\left[\sigma_{\text {abs }, \mathrm{i}}(\lambda)\right]$. The relation between the cross section $\sigma$ and the often used molar decadic coefficient $\epsilon$ is $\epsilon=\sigma N_{\mathrm{A}} /\left[1000 \mathrm{~cm}^{3} \ln (10)\right]$ (dimension: $\mathrm{mole}^{-1}$ $\left.\mathrm{cm}^{-1}\right)$.

The $\sigma_{\mathrm{abs}, \mathrm{M}}(\lambda), \sigma_{\mathrm{abs}, \mathrm{D}}(\lambda), \sigma_{\mathrm{em}, \mathrm{M}}(\lambda)$ and $\sigma_{\mathrm{em}, \mathrm{D}}(\lambda)$ spectra of rhodamine $6 \mathrm{G}$ in methanol and water are depicted in figs. 7 and 8 , respectively. The absorption cross-section spectra are taken from [13] and [16]. The stimulated emission cross-section spectra of the closely spaced pairs of rhoda- mine $6 \mathrm{G}$ in methanol and of the stable groundstate dimers of rhodamine $6 \mathrm{G}$ in water are strongly broadened and shifted to longer wavelengths compared to the monomer spectra. The $\sigma_{\mathrm{em}, \mathrm{D}}(\lambda)$ spectrum of rhodamine $6 \mathrm{G}$ in water is not very accurate because of the inaccurate determination of $E_{\mathrm{D}}(\lambda)$ around the emission peak. The total integrated emission cross sections of the monomers and the molecules in dimers are of similar strength [rhodamine $6 \mathrm{G}$ in methanol:

$$
\begin{aligned}
& \int_{\mathrm{em}} \sigma_{\mathrm{em}, \mathrm{M}}(\tilde{\nu}) \mathrm{d} \tilde{\nu}=5.7 \times 10^{-13} \mathrm{~cm} ; \\
& \int_{\mathrm{em}} \sigma_{\mathrm{em}, \mathrm{D}}(\tilde{\nu}) \mathrm{d} \tilde{\nu}=4.2 \times 10^{-13} \mathrm{~cm} ;
\end{aligned}
$$

rhodamine $6 \mathrm{G}$ in water:

$$
\begin{aligned}
& \int_{\mathrm{em}} \sigma_{\mathrm{em}, \mathrm{M}}(\tilde{\nu}) \mathrm{d} \tilde{\nu}=4.4 \times 10^{-13} \mathrm{~cm} ; \\
& \left.\int_{\mathrm{em}} \sigma_{\mathrm{em}, \mathrm{D}}(\tilde{\nu}) \mathrm{d} \tilde{\nu}=5 \times 10^{-13} \mathrm{~cm}\right]
\end{aligned}
$$

\section{Dimer fluorescence lifetime}

The dimer fluorescence lifetimes may be estimated from the radiative lifetimes $\tau_{\text {rad,D }}$ (eq. (13)) and the quantum efficiencies $q_{\mathrm{D}}$ by use of the relation

$\tau_{\mathrm{D}}=q_{\mathrm{D}} \tau_{\mathrm{rad}, \mathrm{D}} \cdot$

The experimental results give $\tau_{\mathrm{D}}=3.9$ ps for rhodamine $6 \mathrm{G}$ in methanol $\left(q_{\mathrm{D}}=8.5 \times 10^{-4}\right.$, $\tau_{\text {rad,D }}=4.6 \mathrm{~ns}$ ) and $\tau_{\mathrm{D}}=2.2 \mathrm{ps}$ for rhodamine $6 \mathrm{G}$ in water $\left(q_{\mathrm{D}}=6 \times 10^{-4}, \tau_{\mathrm{rad}, \mathrm{D}}=3.6 \mathrm{~ns}\right)$. In case of rhodamine $6 \mathrm{G}$ in methanol the measured fluorescence lifetime $\tau_{\mathrm{F}}$ at $0.4 \mathrm{~mol} / \mathrm{l}$ (fig. 4, [9]) agrees within the error bars with $\tau_{\mathrm{D}}$. In case of rhodamine $6 \mathrm{G}$ in water the monomer fluorescence still dominates at the solubility limit $(C=0.027 \mathrm{~mol} / 1$, $\left.\tau_{\mathrm{F}} \simeq 150 \mathrm{ps}\right)$ and $\tau_{\mathrm{F}}$ remains considerably longer than $\tau_{D}$. It should be noted in passing that the monomer fluorescence lifetime $\tau_{M}$ decreases less steeply with concentration than $q_{\mathrm{M}}$ since $q_{\mathrm{M}}$ is proportional to the mole fraction $x_{M}=1-x_{D}$ (eq. (10)) while $\tau_{M}$ is independent of this factor. 


\section{Interpretation of dimer spectra}

The absorption and emission cross-section spectra may be qualitatively interpreted with the aid of the configuration diagrams of fig. 9. Figure 9a represents the potential energy surface diagram (energy versus intra-molecular configuration coordinate) for a monomer. The $S_{0}$ and the $S_{1}$ band is shown. The dominant vibrational breathing mode in the $\mathrm{S}_{0}\left(v^{\prime \prime}=1\right)$ and the $\mathrm{S}_{1}$ band $\left(v^{\prime}=1\right)$ is indicated (vibrational energy $\simeq 1500 \mathrm{~cm}^{-1}$ ). The hatched areas mark the regions of Franck-Condon overlap for the absorption and the emission. The Franck-Condon shift $\Delta_{M}$ is responsible for the vibronic structure of the monomer absorption and emission spectrum $[12,14,16]$. In the absorption process the $\mathrm{S}_{0}\left(v^{\prime \prime}=0\right) \rightarrow \mathrm{S}_{1}\left(v^{\prime}=0\right)$ Franck-Condon factor dominates over the $\mathrm{S}_{0}\left(v^{\prime \prime}\right.$ $=0) \rightarrow \mathrm{S}_{1}\left(v^{\prime}=1\right)$ Franck-Condon factor. For the emission the $\mathrm{S}_{1}\left(v^{\prime}=0\right) \rightarrow \mathrm{S}_{0}\left(v^{\prime \prime}=0\right)$ transition dominates over the $\mathrm{S}_{1}\left(v^{\prime}=0\right) \rightarrow \mathrm{S}_{0}\left(v^{\prime \prime}=1\right)$ transition.

The configuration diagrams of the two dye molecules in a dimer (stable ground-state dimer or closely spaced pair) are illustrated in fig. 9b. Compared to the monomer the potential energy surfaces are somewhat lowered to indicate the binding between both molecules. The $\mathrm{S}_{1}$-state lowering is shown a little bit larger than the $\mathrm{S}_{0}$-state lowering to account for the long-wavelength shift of the dimer absorption cross-section spectra. The energy levels of both molecules in the dimer are somewhat different (exciton splitting [26-28]) due to mutual interaction (Pauli exclusion principle). The Franck-Condon shifts, $\Delta_{\mathrm{D} 1}$ and $\Delta_{\mathrm{D} 2}$, of both molecules are assumed to be larger than the Franck-Condon shift $\Delta_{M}$ of an undisturbed monomer.

The enlarged Franck-Condon shifts allow to explain the observed shape of the dimer absorption and emission cross-section spectra of figs. 7 and $8[13,26,29,30]$ : (i) In the absorption process the $\mathrm{S}_{0}\left(v^{\prime \prime}=0\right) \rightarrow \mathrm{S}_{1}\left(v^{\prime}=1\right)$ transition gains importance (enlarged Franck-Condon overlap integral, see hatched regions $/ / / /$ ) compared to the $\mathrm{S}_{0}\left(v^{\prime \prime}=0\right) \rightarrow \mathrm{S}_{1}\left(v^{\prime}=0\right)$ transition which dominates for the monomers. In case of rhodamine $6 \mathrm{G}$ in water (stable ground-state dimer) the $\mathrm{S}_{0}\left(v^{\prime \prime}=0\right) \rightarrow \mathrm{S}_{1}\left(v^{\prime}=1\right)$ absorption becomes larger than the $\mathrm{S}_{0}\left(v^{\prime \prime}=0\right) \rightarrow \mathrm{S}_{1}\left(v^{\prime}=0\right)$ absorption (absorption peaks at $500 \mathrm{~nm}$, fig. 8). For rhodamine $6 \mathrm{G}$ in methanol (closely spaced pairs) the Franck-condon overlap integrals are approximately equal for the $\mathrm{S}_{0}\left(v^{\prime \prime}=0\right) \rightarrow \mathrm{S}_{1}\left(v^{\prime}=1\right)$ and

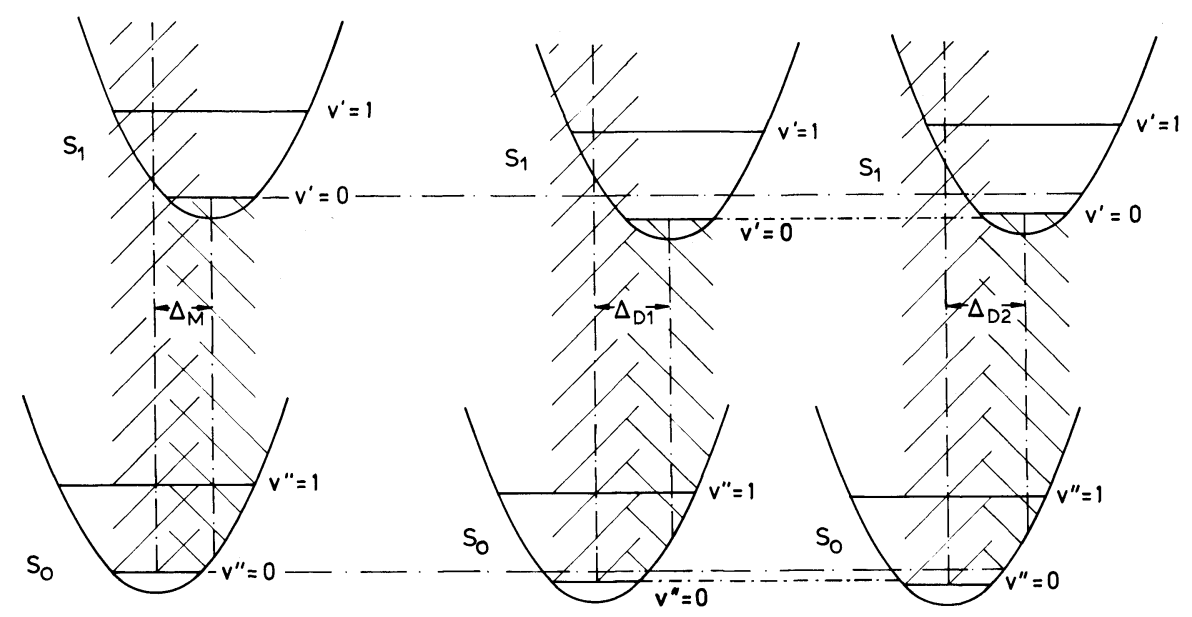

(a)

(b)

Fig. 9. Schematic configuration coordinate diagrams for a monomer (a), and for the two dye molecules forming a dimer (b). The vertical coordinate is energy, the horizontal coordinate is an intra-molecular distance. Parameters are explained in the text. 
the $\mathrm{S}_{0}\left(v^{\prime \prime}=0\right) \rightarrow \mathrm{S}_{1}\left(v^{\prime}=0\right)$ transition resulting in the double peaked absorption spectrum of fig. 7 . (ii) For the emission process the enlarged Franck-Condon shift $\left(\Delta_{\mathrm{D} 1}, \Delta_{\mathrm{D} 2}\right)$ leads to an extended long-wavelength Franck-Condon overlap (see hatched regions $\backslash \backslash \backslash \backslash$ ). Consequently the stable ground-state dimer stimulated emission cross-section spectrum (fig. 8 , rhodamine $6 \mathrm{G}$ in water) and the closely spaced pair stimulated emission cross-section spectrum (fig. 7, rhodamine $6 \mathrm{G}$ in methanol) extend further to the long-wavelength region than the monomer spectra.

The absorption and emission spectra of figs. 7 and 8 give the overall behaviour of both molecules in the dimer (average over both molecules in dimers). Different Franck-Condon shifts for monomers and dimers were previously assumed for the interpretation of dimer spectra in $[13,26,29,30]$. The applied qualitative dimer model of fig. $9 \mathrm{~b}$ is consistent with (i) the approximately constant energy separation between absorption peak and vibronic shoulder of the monomer, between the two absorption peaks in the closely spaced pairs, and between the long-wavelength absorption shoulder and the short-wavelength absorption peak of the stable ground-state dimers, (ii) the longwavelength extension of the dimer fluorescence compared to the monomer fluorescence, (iii) the strong total integrated dimer emission cross section, and (iv) the possibility to observe fluorescence emission despite the short dimer fluorescence lifetime (electric dipole allowed transition from relaxed excited state with radiative lifetime $\tau_{\text {rad,D }}$ in the nanosecond region). Unfortunately fluorescence polarization spectroscopy cannot be used to interpret the dimer spectra of rhodamine $6 \mathrm{G}$ in methanol and water because of the fast energy transfer depolarization (see above).

\section{Conclusions}

The concentration-dependent fluorescence emission of rhodamine $6 \mathrm{G}$ in methanol and in water was analyzed. In methanol ground-state dimer formation is unstable (dimer binding energy $E_{\mathrm{B}}<k T$ ) and closely spaced pairs dominate the fluorescence behaviour at high concentrations. In water stable ground-state dimers are formed $\left(E_{\mathrm{B}}\right.$ $>k T)$. The solubility is limited to $C<0.027$ $\mathrm{mol} / \mathrm{l}$. In both solvents the fluorescence quantum efficiency is quenched by Förster-type energy transfer to weakly fluorescing dimers (closely spaced pairs in case of methanol, ground-state dimers in case of water). From the measured fluorescence spectra the monomeric and dimeric contributions to the fluorescence quantum distribution and to the fluorescence quantum efficiency were resolved and the stimulated emission crosssection spectra of the dimers were determined. The difference between the monomeric and dimeric absorption and stimulated emission crosssection spectra indicates an enlarged Franck-Condon shift of the dimers compared to the monomers.

\section{Acknowledgements}

The authors thank the Deutsche Forschungsgemeinschaft for financial support and the Rechenzentrum of the University for computer time put at their disposal.

\section{References}

[1] Th. Förster and E. König, Zeitschrift für Elektrochemie 61 (1957) 344.

[2] G.S. Levinson, W.T. Simpson and W. Curtis, J. Am. Chem. Soc. 79 (1957) 4314.

[3] R.W. Chambers, T. Kajiwara and D.R. Kearns, J. Phys. Chem. 78 (1974) 380.

[4] R.R. Alfano, S.L. Shapiro and W. Yu, Opt. Commun. 7 (1973) 191.

[5] F. Fink, E. Klose, K. Teuchner and S. Dähne, Chem. Phys. Lett. 45 (1977) 548.

[6] K.A. Selanger, J. Falnes and T. Sikkeland, J. Phys. Chem. 81 (1977) 1960.

[7] D.R. Lutz, K.A. Nelson, C.R. Gochanour and M.D. Fayer, Chem. Phys. 58 (1981) 325.

[8] A.L. Smirl, J.B. Clark, E.W. Van Stryland and B.R. Russell, J. Chem. Phys. 77 (1982) 631.

[9] A. Penzkofer and Y. Lu, Chem. Phys. 103 (1986) 399.

[10] A. Budó and I. Ketskeméty, J. Chem. Phys. 25 (1956) 595.

[11] P.R. Hammond, J. Chem. Phys. 70 (1979) 3884.

[12] C.A. Parker, Photoluminescence of Solutions (Elsevier, Amsterdam, 1968).

[13] Y. Lu and A. Penzkofer, Chem. Phys. 107 (1986) 175. 
[14] Th. Förster, Fluoreszenz Organischer Verbindungen (Vandenhoeck und Ruprecht, Göttingen, 1951).

[15] B. Kopainsky, J.K. Hallermeier and W. Kaiser, Chem. Phys. Lett. 87 (1982) 7.

[16] J.E. Selwyn and J.I. Steinfeld, J. Chem. Phys. 76 (1972) 762.

[17] A. Penzkofer, Appl. Phys. B 40 (1986) 85.

[18] F. Dörr, Angewandte Chemie 78 (1966) 457.

[19] E.D. Cehelnik, K.D. Mielenz and R.A. Velapoldi, J. Res. Nat. Bureau of Standards 79A (1975) 1.

[20] H.J. Eichler, U. Klein and D. Langhans, Chem. Phys. Lett. 67 (1979) 21.

[21] R.W. Wijnaendts van Resandt and L. DeMaeyer, Chem. Phys. Lett. 78 (1981) 219

[22] K. Berndt, H. Dürr and D. Palme, Opt. Commun. 42 (1982) 419.
[23] O.G. Peterson, J.P. Webb, W.C. McColgin and J.H. Eberly, J. Appl. Phys. 42 (1971) 1917.

[24] S.J. Strickler and R.A. Berg, J. Chem. Phys. 37 (1962) 814.

[25] J.B. Birks and D.J. Dyson, Proc. Roy. Soc. London A 275 (1963) 135.

[26] M. Pope and C.E. Swenberg, Electrical Processes in Organic Crystals (Clarendon Press, Oxford, 1982).

[27] M. Kasha, in: Spectroscopy of the Excited State, ed. B. DiBartolo (Plenum Press, New York, 1976) p. 337.

[28] N. Karl, in: Festkörperprobleme, Vol. 14 (Vieweg, Braunschweig, 1974) p. 261.

[29] V. Zanker, M. Held and H. Rammensee, Z. Naturforschg. 14b (1959) 789.

[30] E.A. Chandross and J. Ferguson, J. Chem. Phys. 45 (1966) 4532 . 